\title{
The Patched gene is epigenetically regulated in ovarian dermoids and fibromas, but not in basocellular carcinomas
}

\author{
MAJA CRETNIK ${ }^{1}$, VESNA MUSANI ${ }^{1}$, SLAVKO ORESKOVIC ${ }^{2}$, \\ DINKO LEOVIC ${ }^{3}$ and SONJA LEVANAT ${ }^{1}$ \\ ${ }^{1}$ Division of Molecular Medicine, Rudjer Boskovic Institute, 10002 Zagreb; ${ }^{2}$ Department of Obstetrics \\ and Gynecology, Clinical Hospital Petrova, School of Medicine, University of Zagreb, 10002 Zagreb; \\ ${ }^{3}$ Department of Maxillofacial Surgery, Clinical Hospital Osijek, 31000 Osijek, Croatia
}

Received January 30, 2007; Accepted March 5, 2007

\begin{abstract}
The Hedgehog/Patched signaling pathway plays a prominent role during mammalian development but it is also involved in oncogenic transformation. We investigated the methylation status of the Patched promotor in a set of basocellular carcinomas of the skin and ovarian tumors as an alternative to mutational causes of the pathway deregulation. Our aim was to define a possible role of genetic and/or epigenetic mechanisms of Hedgehog/Patched signal transduction in the development of these tumors. Bisulfite-converted DNA from tumors and from matched healthy tissue was amplified by a specific PCR and the CpG-rich regions of the Patched promoter were sequenced. Two promoter regions showed statistically significant hypermethylation compared to healthy controls in ovarian tumors; more significantly in the region in the vicinity of Gli1-binding sites and less significantly in the region containing the ATG codon. But, in basocellular carcinomas of the skin we observed no difference in methylation, suggesting different mechanisms of neoplasia in these tumors.
\end{abstract}

\section{Introduction}

Research on the Hedgehog/Patched pathway involvement in a number of common human cancers (1) relies heavily on investigations of the pathway malfunctioning in less frequent or barely malignant neoplasia related to Gorlin syndrome. In this manuscript we present the results of an investigation aimed at evaluating the potential of Patched hypermethylation as an

Correspondence to: Dr Sonja Levanat, Division of Molecular Medicine, Rudjer Boskovic Institute, Bijenicka 54, HR 10002 Zagreb, Croatia

E-mail: levanat@irb.hr

Key words: methylation, patched promoter, Hh-Gli signaling, tumors alternative to mutational causes of the pathway deregulation in different tumors, indicating two unrelated events. The choice of tumors came from previous investigations on tumors involved in Gorlin syndrome.

Gorlin syndrome or Basal Cell Nevus Syndrome (BCNS) is a rare heritable autosomal dominant disorder characterized by developmental malformations and a predisposition to basocellular carcinoma (BCC), odontogenic keratocysts (OKCs), ovarian fibroma, medulloblastoma and other neoplasia. Frequent loss of heterozygosity (LOH) at the $9 \mathrm{q} 22.3$ locus in BCNS neoplastic formations facilitated the cloning of the Patched (PTCH) gene (2) a tumor suppressor (also known as PTCH1), and a human homologue of the Drosophila gene Patched. It also led to the explanation that the syndrome is caused by constitutional hemizygous alteration of the $\mathrm{PTCH}$ gene, confirming Knudson's two-hit hypothesis (3) that total inactivation of the gene (enabling neoplastic proliferation) can be more easily achieved by a somatic hit on the remaining wild-type allele in BCNS than by inactivation of both alleles in the general population.

Subsequent investigations of typical BCNS neoplasia in the general population, BCC in particular, revealed that they could also be caused by activating mutations of the Smoothened gene $(S M O)$, as an alternative to $P T C H$ inactivation. This contributed to a further understanding of the Hedgehog/Patched (HH/PTC) pathway, also called the $\mathrm{HH} / \mathrm{GLI}$ pathway, or SHH/PTCH pathway in mammals, due to the prevailing role of the Sonic Hedgehog ( $\mathrm{SHH}$ ) mammalian homologue of HH. This pathway has important functions during development, when its activation is normally initiated by the secreted protein Shh, a ligand that binds to its trans-membrane receptor Ptch. When this happens, Ptch relieves its repression of the co-receptor Smo, also a membrane protein. Smo then activates a downstream cascade that leads to the translocation of the active form of the transcription factor Gli1 to the nucleus (4). Many of Gli1 targets are associated with cell proliferation, indicating that this proto-oncogene can induce cell transformation through multiple pathways (1). In addition, PTCH itself is also included among the Gli targets, and auto-regulation of the SHH/PTCH pathway is normally achieved by increased production of Ptch protein. 
The role of $P T C H$ in maintaining and restoring the repression of Smo crucially depends on the ability of the gene to produce functional Ptch protein and to increase production of Ptch protein after the pathway activation. Various PTCH mutations causing C-terminal truncation of Ptch protein, after which the latter can no longer repress Smo, are well-documented mechanisms of the SHH/PTCH pathway deregulation (5). Whereas Ptch haploinsufficiency caused by the constitutive mutations of one PTCH allele in BCNS is hypothesized to be the likely cause for developmental malformations in the syndrome, tumors typical of BCNS generally require full loss of the $P T C H$ regulatory function (unless they are caused by alterations of other pathway genes). In many of these tumors, Ptch disabling mutations have been found on both alleles, but in others no alterations in $P T C H$ coding sequence have been seen despite indications that the gene is involved in their development (such as the $\mathrm{LOH}$ at the 9q22.3 locus). Therefore, the interest of research has been expanded to the $\mathrm{PTCH}$ promoter region as another potentially weak spot, where two Gli-binding sites mediate downstream pathway regulation through $P T C H$ expression.

In our previous investigations of BCNS-related disorders, OKCs in particular, we detected $\mathrm{LOH}$ at the PTCH locus in dentigerous cysts as well, which led us to hypothesize that the PTCH gene may be involved in developmental cysts in general (6). The possibility that such mild disorders with low incidence in the general population may be related to the PTCH gene, but not associated with BCNS, can perhaps be explained by the very low prevalence of this syndrome.

Due to similar expression levels of pathway genes, we have included ovarian dermoids in our more recent investigations of ovarian fibroma (7). The latter are very strongly associated with BCNS and therefore expected to be largely caused by PTCH alterations: the incidence of ovarian fibroma in the syndrome patients is several times greater than in the general female population, and this relative increase is comparable to or greater than that of $\mathrm{BCCs}$, the most common syndrome neoplasia.

Although increased expression levels of several SHH/ PTCH pathway genes in a number of tumors obtained from the general population have provided additional indication of the pathway involvement in both ovarian fibroma and dermoids (8), direct sequencing of $P T C H$ exons revealed no mutations. Since it would be too unlikely that the $P T C H$ gene is not involved at least in some fibroma, we have proceeded to analyze the $P T C H$ promoter, focusing on the methylation of the region. In order to not exclude other tumors where $P T C H$ mutations are very common, we examined BCCs in the same way. Inactivating mutations of $P T C H$ in sporadic BCCs and, consequently, different levels of expression of members of the Hh-Gli signaling pathway have been reported (9), but the PTCH promoter status has not yet been observed.

Changes in the patterns of DNA methylation have been correlated with a number of different processes in mammals (10). However, more relevant for this investigation is the phenomenon of hypermethylation of the gene promoter (11). It has been established that $\mathrm{CpG}$ island methylation in the promoter region can inactivate tumor suppressor genes in human cancers, and that such $\mathrm{CpG}$ sites often show an increased mutation rate $(12,13)$. Also, promoter hypermethylation of several tumor suppressor genes has already been reported in primary ovarian carcinoma (14).

We examined the methylation status of the PTCH promoter in different set of tumors; basocellular carcinomas, ovarian fibromas and ovarian dermoids.

\section{Materials and methods}

Samples and procedures. All samples were obtained from the Department of Obstetrics and Gynecology, School of Medicine, University of Zagreb; Clinical Hospital Petrova, Zagreb; and from the Department of Maxillofacial Surgery, Clinical Hospital Osijek, Osijek, Croatia. During surgery the samples were excised for pathohistological examination (by a pathologist), and for molecular analysis. For molecular analysis we used cyst lining in the case of ovarian dermoids, and stromal tissue in the case of ovarian fibroma and basocellular carcinomas of the skin. Both dermoids and fibroma can be found anywhere in the body, but since all our samples were taken from ovaries, stromal tissue from healthy ovaries was used as a control for both tumor types. As a control for basocellular carcinomas we used healthy skin samples. Samples were dissected with a scalpel and conserved in culture medium or in RNA Later solution for DNA and RNA isolation. The samples were stripped of identifiers, could not be traced back to patients and were collected with consideration to all necessary ethical and legal requirements.

The tissue samples were denoted as follows: F, ovarian fibroma; D, ovarian dermoids; NO, normal ovaries; B, basocellular carcinomas of the skin; and NS, normal skin. Additional labels ' $a$ ' and ' $b$ ' were introduced when two different samples were taken from the same person (e.g. NO1a and NO1b).

Methylation of a promoter sequence was determined using bisulfite genomic sequencing method (15) which is based on chemical alteration of DNA where sodium bisulfite converts unmethylated cytosines into uracils, but 5-methylcytosine remains unaltered.

Before bisulfite conversion, genomic DNA was cut with restriction enzyme SalI, because it does not cut within the $P T C H$ promoter region, neither the original sequence nor the converted one. After bisulfite conversion, the $\mathrm{CpG}$-rich region of interest was amplified by specifically designed primers.

DNA extraction. DNA from all samples was digested in buffer (0.9\% Tween-20, 0.9\% Triton X-100, 5 mM EDTA, $2 \mathrm{mM}$ DTT, $10 \mathrm{mM}$ TrisHCl $\mathrm{pH} 7.5$ ) and proteinase $\mathrm{K}$ $(20 \mu \mathrm{g} / \mathrm{ml})$ overnight at $55^{\circ} \mathrm{C}$ and extracted by the standard phenol-chloroform method.

Bisulfite modification. DNA $(2 \mu \mathrm{g})$ was cut with restriction enzyme SalI. Restriction was performed overnight at $37^{\circ} \mathrm{C}$. The following day the volume was adjusted to a total of $90 \mu 1$, and denatured with $\mathrm{NaOH}$ (final concentration, $0.3 \mathrm{M}$ ) for $20 \mathrm{~min}$ at $42^{\circ} \mathrm{C}$. A bisulfite and hydroquinone solution was freshly prepared $(2.5 \mathrm{M}$ sodium metabisulfite, $0.001 \mathrm{M}$ hydroquinone, and $0.4 \mathrm{M} \mathrm{NaOH}$ ), dissolved without vigorous shaking and filtered through a $0.45-\mu \mathrm{m}$ filter. This solution (1200 $\mu 1)$ was added directly to the denatured DNA and incubated overnight at $55^{\circ} \mathrm{C}$ in the dark. 
DNA was then purified using Promega Wizard DNA purification kit (Madison, WI, USA), desulfonated with $\mathrm{NaOH}$ (final concentration, $0.3 \mathrm{M}$ ) at $37^{\circ} \mathrm{C}$ for $20 \mathrm{~min}$, neutralized and precipitated overnight with ammonium acetate and carrier tRNA and ethanol. Converted DNA was ready for further PCR amplification.

Conversion efficiency. We verified the conversion efficiency by examining the conversion of non-CpG cytosines into uraciles. The original protocol suggested $4 \mathrm{~h}$ of incubation, but it gave $41 \%$ non-converted cytosines. Doubling the incubation to $8 \mathrm{~h}$ reduced the non-converted cytosines to $36 \%$. Finally, overnight incubation (16-18 h) provided adequate efficiency, with at most $8 \%$ of the non-CpG cytosines not completely converted to thymines (and these were located mostly at the ends of sequences). This $8 \%$ conversion inefficiency limit for non-CpG cytosines was maintained for all samples, both normal and tumor $(>72$ sequences), and for most of the samples the conversion inefficiency limit was considerably lower ( 4-5\%). Sequences with non-CpG conversion inefficiency $>8 \%$ were discarded.

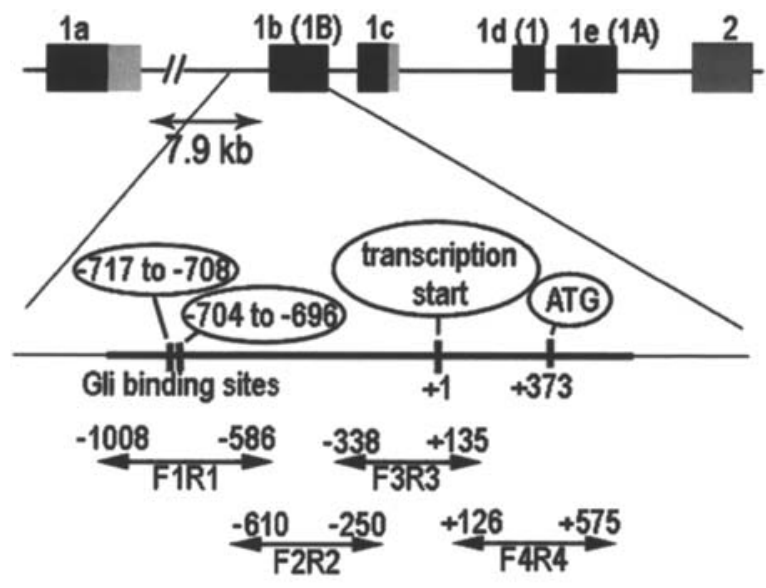

Figure 1. Position of the Patched gene promoter and the first two exons. Alternative forms of exon 1 (a, b, c, d, and e) are marked according to Nagao et al (16), and in parentheses according to Agren et al (4). The promoter is located in the $5^{\prime}$ region upstream of exon $1 \mathrm{~b}$ and encompasses whole exon 1b. It consists of a CpG-rich region of 1593 bp containing 163 CpG sites. The positions of two Gli1-binding sites, transcription start, and ATG codon are marked. Four sets of primers were designed to amplify both methylated and unmethylated DNA of the CpG-rich region.

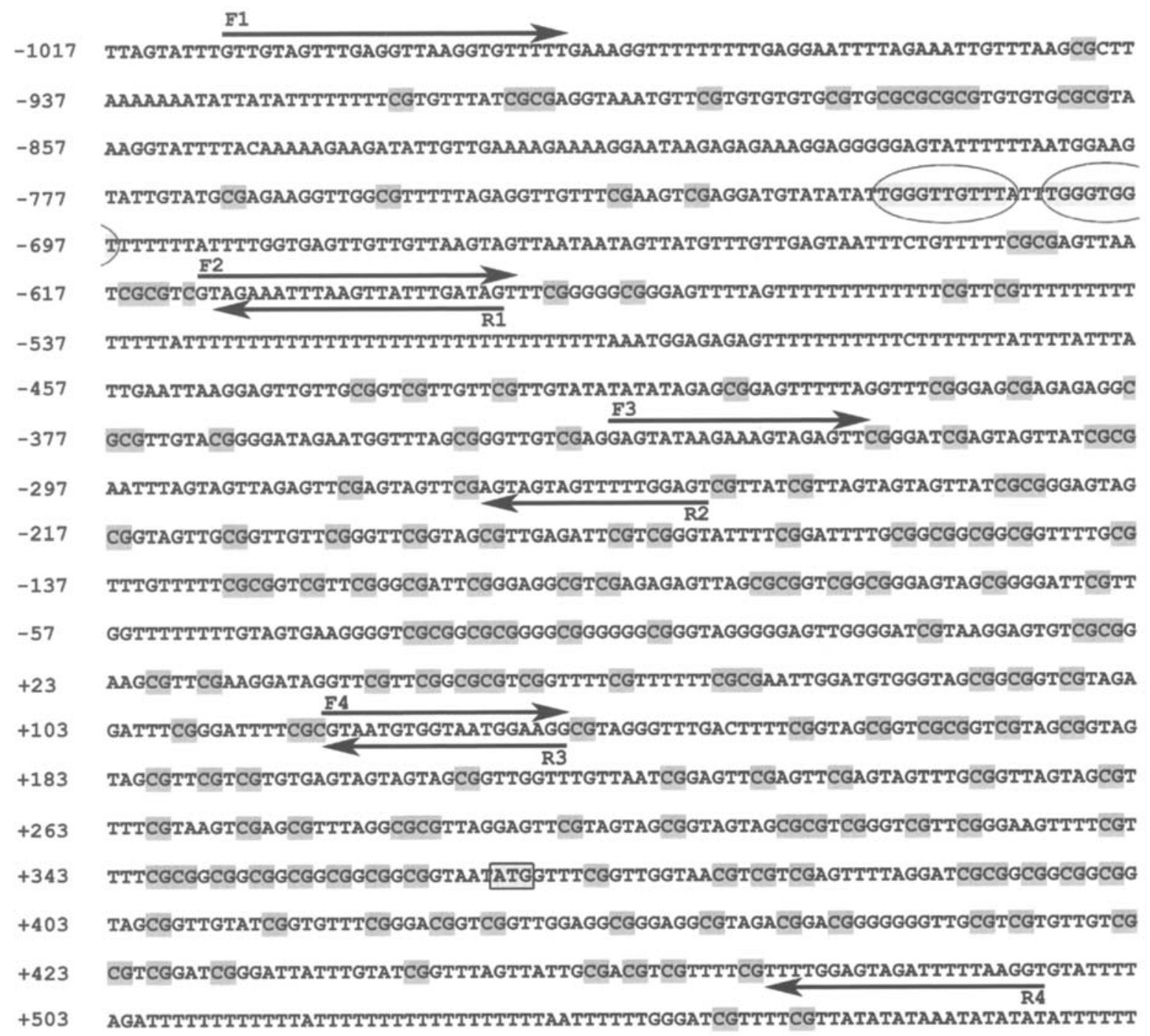

Figure 2. Hypothetical sequence of the analyzed region after bisulfite conversion in which all non-CpG cytosines were replaced with thymines, but cytosines within the $\mathrm{CpG}$ sites remained as cytosines. All CpG islands are shaded; primers of the four sub-regions are indicated by forward and reverse arrows; and Glibinding sites are marked with circles and ATG is in a square. 


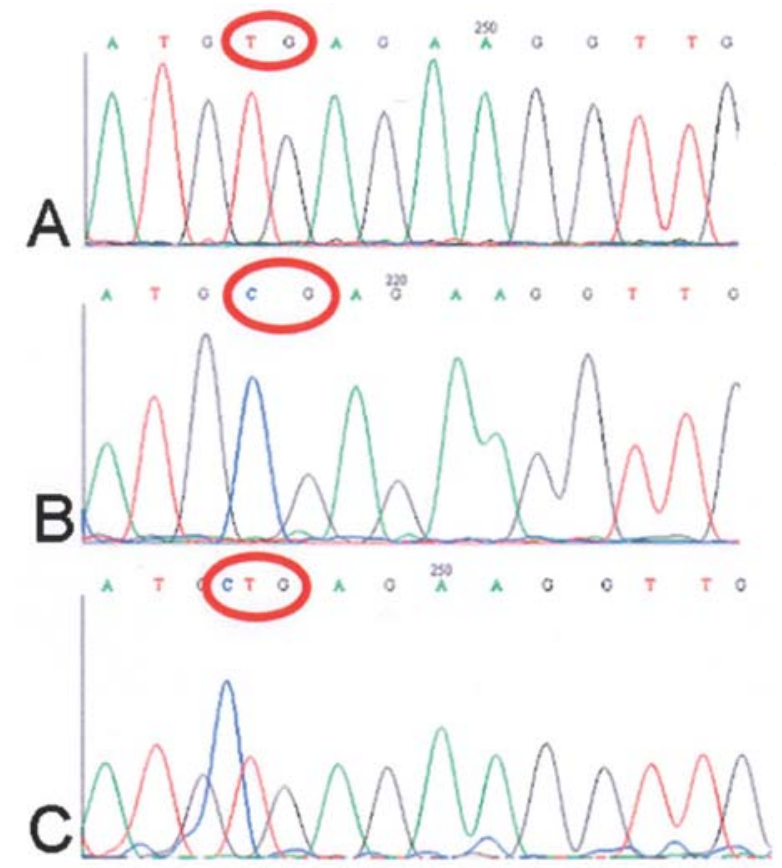

Figure 3. Sequence analysis: unmethylated section of NO 2a (A), methylated section of F1 (B), and partly methylated (heterozygous) section of D4 (C). The same $\mathrm{CpG}$ site is circled in red in all three pictures and corresponds to $\mathrm{CpG}$ site number 13 in the sequence.

Primer design and PCR amplification. The PTCH promoter sequence was determined according to NCBI database (Gene ID no. 5727) and Agren et al (4). Position of the CpG islandrich promoter region selected for analysis $(-1008$ to +575$)$ is schematically shown in Fig. 1, relative to alternative exon 1 sequences (16). It encompasses two Gli1-binding sites and the transcription start at +1 with ATG signal at +373 . Agren et al (4) showed that only the second Gli-binding site, on position -704 to -696 , is fully functional. Details of the selected promoter region are shown in Fig. 2, which presents the hypothetical sequence after bisulfite conversion of all non$\mathrm{CpG}$ cytosines (they are replaced with thymines) in order to facilitate recognition of CpGs and their methylation status. For amplification, the promoter was divided into four subregions, and 4 sets of primers were designed, avoiding CpGs to ensure that all would be amplified, whether methylated or not. The positions of the primers and segments that they cover are marked in both Figs. 1 and 2. Finally, sequencing was performed to compare the number of methylated vs. unmethylated CpGs.

Primer sequences were as follows: PR1 forward, 5'-GTT GTA GTT TGA GGT TAA GGT GTT TTT-3' and reverse, 5'-CTA TCA AAT AAC TTA AAT TTC TA-3'; PR2 forward, 5'-GTA GAA TTT AAG TTA TTT GAT AGT-3' and reverse, 5'-CT CCA AAA ACT ACT ACT-3'; PR3 forward, 5'-GAG TAT AAG AAA GTA GAG TT-3' and reverse, 5'-CCT TCC ATT ACC ACA TTA C-3'; PR4 forward, 5'-GTA ATG TGG TAA TGG AAG G-3' and reverse, 5'-CCT TAA AAA TCT ACT CCA AAA-3'. Primer pair PR1 forward-reverse produces a 422-bp-sized product containing Gli binding sites, and it is denoted as region 1, position from -1008 to -586 . Primer pair PR2 forwardreverse produces a 360-bp segment (region 2, -610 to -250),

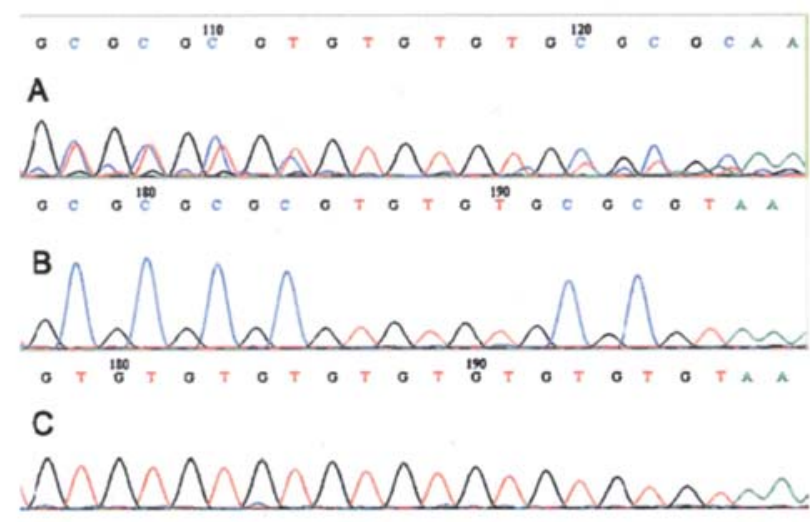

Figure 4. Sequence of a heterozygous sample D3 after direct sequencing of a PCR product (A), and sequences of the same sample obtained after the subcloning of PCR products into bacterial plasmid and sequencing of plasmid DNA from each colony separately (B and C). It is clearly evident that one of the alleles is methylated (B), and the other unmethylated (C). The CpG sites shown are 7-12.

whereas primer pair PR3 forward-reverse produces a 473-bpsized product (region $3,-338$ to +135 ). Primer pair PR4 forward-reverse amplifies a 449-bp-sized segment containing the start codon ATG (region $4,+126$ to +575 ).

Converted DNA ( $1 \mu \mathrm{l})$ was used in a $25-\mu 1$ PCR reaction, with AmpliTaq Gold enzyme at the following conditions: $95^{\circ} \mathrm{C}$ for $10 \mathrm{~min}$, and 45 times $95^{\circ} \mathrm{C}$ for $1 \mathrm{~min}, 50^{\circ} \mathrm{C}$ for $2 \mathrm{~min}$, $70^{\circ} \mathrm{C}$ for $2 \mathrm{~min}$ and then $68^{\circ} \mathrm{C}$ for $10 \mathrm{~min}$. The PCR product $(1 \mu \mathrm{l})$ was used as a template for a second amplification in a $50-\mu 1$ PCR reaction, with the same cycling conditions. The PCR product was excised from the $1 \%$ agarose gel and purified for the next step (Qiagen Gel extraction kit).

Subcloning of PCR fragments. Amplified PCR fragments were subcloned by the method of Marchuk et al (18) using plasmid vector pBluescript $\mathrm{SK}^{+}$(Stratagene, Heidelberg, Germany) and transformed into host E. coli XL1 Blue (Stratagene) for propagation. Plasmid DNA from positive clones was sequenced.

Sequencing. All samples were sequenced by the dideoxy method (17) using BigDye ${ }^{\circledR}$ chemistry with Terminator v.1.1 kit and analyzed on ABI PRISM 310 genetic analyser using DNA sequencing analysis software version 5.1. (Applied Biosystems, Foster City, CA, USA).

Sequences were analyzed using the BioEdit program. Each $\mathrm{CpG}$ site was numbered, examined and determined as: methylated (only $\mathrm{C}$ present), heterozygous (both $\mathrm{C}$ and $\mathrm{T}$ present; one strand was methylated and the other was not), or unmethylated (only T present). Fig. 3 shows the sequence analysis of methylated, unmethylated and partly methylated (heterozygous) samples.

All samples were directly sequenced from a PCR product (Fig. 4A), and for each group of samples at least two PCR products were subcloned into bacterial plasmid and then each colony was sequenced separately (Fig. 4B and C). By parallel checking it was clearly evident when one of the alleles was methylated (Fig. 4B), or unmethylated (Fig. 4C). 

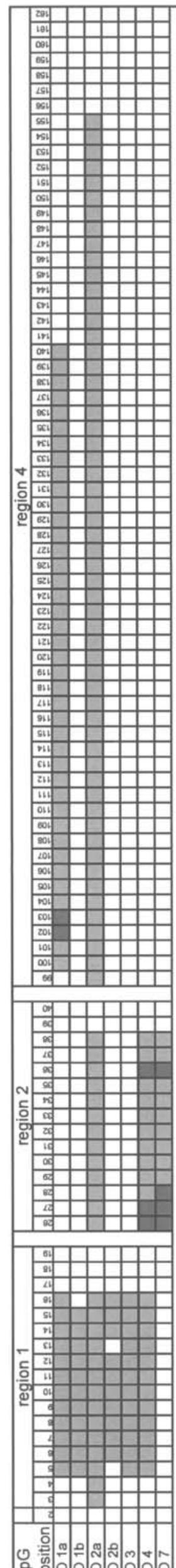
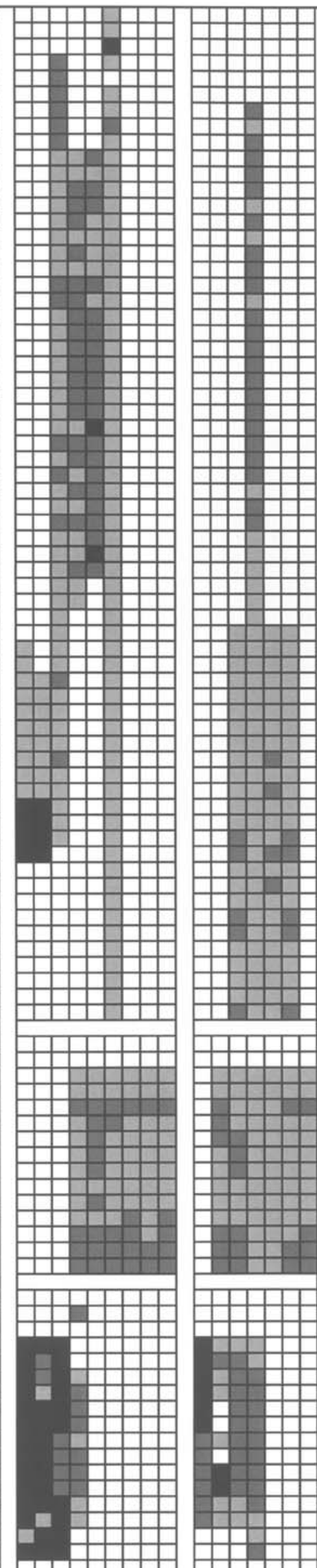
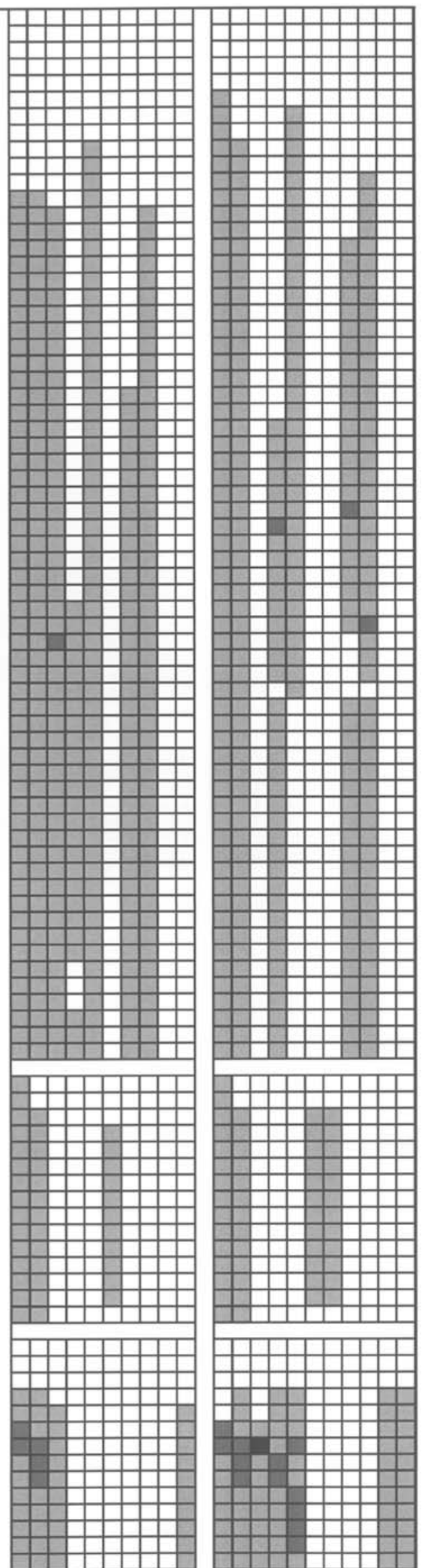

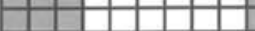

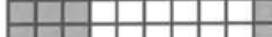

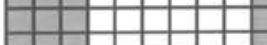

17 献

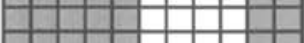

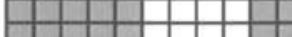

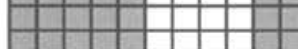

ह

옹

要

声

ํํㅇ

卷

仓ㄹ

क्ष

ن

证

든

会

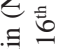

部

도

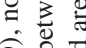

e

융

을

\%

踏

व.

过要

要

它

臣

b

○ิ

s 형

돈

ठठ용

咞

용

啳

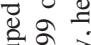

들

记

政

तi

है

可㻤

.

ह 일

우

造

ज语

을 일

$\Xi$.

을음

동

음

붕응

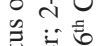

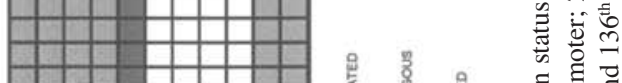

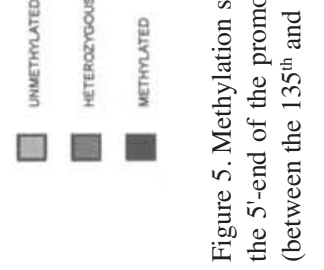


Screening difficulties. We attempted to analyze 50 different samples. The analysis was partly successful for 44 samples (Fig. 5): of 10 fibroma tissues, 9 were successfully sequenced (4 for region 1, 6 for region 2, and 6 for region 4); of 12 dermoids, 7 were successful ( 4 for region 1, 6 for region 2, and 4 for region 4 ); of 15 normal ovary samples, 7 had readable sequences ( 6 for region 1, 3 for region 2 , and 2 for region 4); of 11 basocellular carcinomas of the skin, 7 were successful for region 1, 4 for region 2 and 6 for region 4; and of the 10 normal skin samples, 4 were successful for region 1, 3 for region 2 and 7 for region 4 .

Expression analysis. RNA was isolated from basocellular carcinoma tissue or healthy skin tissue using Trizol reagent (Invitrogen), and additionally purified using High Pure RNA isolation kit (Roche). RNA was reverse transcribed using TaqMan reverse transcription kit (Roche). PCR was performed using the following primers: Arp forward, 5'-GGCACCAT TGAAATCCTGAGTGATGTG-3' and reverse, 5'-TTGCG GACACCCTCCAGGAAGC-3'; Ptch forward, 5'-TCCTCG TGTGCGCTGTCTTCCTTC-3' and reverse, 5'-CGTCAGAA AGGCCAAAGCAACGTGA-3'; Gli1 forward, 5'-GCCGT GTAAAGCTCCAGTGAACACA-3' and reverse, 5'-TCCC ACTTTGAGAGGCCCATAGCAAG-3'. Cycling conditions were $95^{\circ} \mathrm{C}$ for $10 \mathrm{~min}$; 40 times $95^{\circ} \mathrm{C}$ for $30 \mathrm{sec}, 60^{\circ} \mathrm{C}$ for $45 \mathrm{sec}, 72^{\circ} \mathrm{C}$ for $45 \mathrm{sec}$; and then $72^{\circ} \mathrm{C}$ for $7 \mathrm{~min}$. PCR products were separated on a $2 \%$ agarose gel and visualized using Image Master VDS (Pharmacia Biotech.). Arp expression was used as a housekeeping control.

\section{Results}

The methylation status of the $\mathrm{PTCH}$ promoter in ovarian fibroma, ovarian dermoids, BCCs and matched healthy tissue was investigated following bisulfite conversion, specific PCR amplification and sequencing. The promoter region (1593-bp long with $163 \mathrm{CpGs}$ ) was divided into four segments and each of them was sequenced and analyzed separately (Figs. 1 and 2).

Region 1 containing Gli-binding sites. The first part of the promoter, region 1 (-1008 to -586) contains $21 \mathrm{CpG}$ sites and two Gli-binding sites (-717 to -708 and -704 to -696$)$ located between the $16^{\text {th }}$ and the $17^{\text {th }} \mathrm{CpG}$ site. In all samples the sequence was readable only from the $2^{\text {nd }} \mathrm{CpG}$ site on, and we successfully analyzed and sequenced between 10 and 15 CpG sites per sample (Fig. 5).

Average methylation of region 1 in ovarian tumors was $78.88 \%$ of successfully analyzed CpGs $(87.82 \%$ in fibromas and $69.94 \%$ in dermoids), versus $0 \%$ in normal ovaries. These two groups of samples (tumors vs. controls) showed a statistically significant difference by the Student's t-test, $\mathrm{p}=0.0003$ for fibroma and 0.007 for dermoids (Table I). Difference in methylation between ovarian tumors and normal ovaries is most consistent in the vicinity of Gli-binding sites, which are surrounded by a scattered $\mathrm{CpG}$ formation extending from the $13^{\text {th }}$ to the $21^{\text {st }}$ site (first twelve CpGs form a cluster far from Gli sites).

Our analysis (Fig. 5) showed that tumors were mostly methylated near Gli-binding sites, whereas in normal samples there was no methylation at all on the $13^{\text {th }}$ to the $16^{\text {th }}$ CpG site.

Samples F1 and F2, in which $\mathrm{LOH}$ was previously detected for the 9q22.3 region (7), showed on average moderately higher methylation than the rest of the tumors (which had no LOH), but the difference was hardly significant, especially because samples F3 and D1 had similar hypermethylation (Table II).

As an additional control, two samples ('a' and 'b') were taken from the same ovaries. Analysis of such pairs was successful only for region 1, confirming that both samples in each pair had either identical or reasonably similar methylation (Fig. 5).

In contrast to the ovarian and fibroma samples, the methylation status did not differ significantly for BCC samples in relation to healthy skin. BCCs had on average $12.82 \%$ methylated CpGs, while healthy skin showed $8.33 \%$ methylated sites, $\mathrm{p}=0.26$ (Table I).

Region 2. Region 2 covers 21 CpGs (-610 to -250 ), but the analysis was generally successful for 15 islands (the $26^{\text {th }}$ to the $40^{\text {th }}$ ). Average methylation of $\mathrm{CpGs}$ in this region was $17.95 \%$ for normal ovary samples and on average $32.05 \%$ for ovarian and dermoid samples $(39.74 \%$ for fibromas and

Table I. Difference in methylation in tumor tissues (F, fibroma; D, dermoid; B, basocellular carcinoma) and the control as determined by the Student's t-test.

\begin{tabular}{lccrc}
\hline & Samples & $\begin{array}{c}\text { Mean (tumor tissue }) \\
(\% \text { methylation })\end{array}$ & $\begin{array}{c}\text { Mean (control tissue) } \\
\text { (\% methylation) }\end{array}$ & $\begin{array}{c}\text { p-value } \\
\text { Region 1 }\end{array}$ \\
& F & 87.82 & 0.00 & 0.0003 \\
& D & 69.94 & 0.00 & 0.0070 \\
Region 2 & B & 12.82 & 8.33 & 0.2620 \\
& F & 39.74 & 17.94 & 0.0620 \\
Region 4 & D & 24.35 & 17.94 & 0.3150 \\
& F & 0.00 & 0.00 & N/A \\
& D & 38.48 & 2.43 & 0.0110 \\
& B & 23.48 & 2.43 & 0.0090 \\
\hline
\end{tabular}


Table II. Patched promoter methylation data and BCC (B) expression data collated with previous results (7) of the Hedgehog/Patched pathway gene expression in ovarian fibroma (F) and dermoids (D).

\begin{tabular}{|c|c|c|c|c|c|c|}
\hline \multirow[t]{2}{*}{ Sample } & \multicolumn{3}{|c|}{ Expression } & \multicolumn{3}{|c|}{ Methylation (\%) } \\
\hline & $A R P$ & PTCH & GLII & Region 1 & Region 2 & Region 4 \\
\hline $\mathrm{F} 1$ & + & - & + & 92.86 & & 28.57 \\
\hline $\mathrm{F} 2$ & + & - & - & 85.71 & & 33.33 \\
\hline F3 & + & - & + & 100.00 & & 26.00 \\
\hline $\mathrm{F} 4$ & + & + & - & 72.73 & 30.77 & 62.07 \\
\hline F5 & + & - & - & & 69.23 & 77.78 \\
\hline F6 & + & - & + & & 38.46 & 3.17 \\
\hline D1 & + & - & + & 100.00 & & \\
\hline D2 & + & - & + & 33.33 & 46.15 & \\
\hline D3 & + & - & + & 75.00 & 46.15 & 20.00 \\
\hline D4 & + & + & - & 71.43 & 0.00 & 37.93 \\
\hline D5 & + & - & + & & 0.00 & 16.00 \\
\hline B1 & + & + & + & 15.38 & 0.00 & 0.00 \\
\hline B2 & + & - & - & 20.00 & 0.00 & 0.00 \\
\hline B3 & + & + & + & 7.69 & & \\
\hline B4 & + & + & + & 13.33 & & 2.63 \\
\hline B5 & + & + & + & 33.33 & & 0.00 \\
\hline B6 & + & - & - & & 0.00 & \\
\hline B7 & + & + & + & & 0.00 & \\
\hline B8 & + & - & + & & & 2.17 \\
\hline B9 & + & - & - & & & 1.89 \\
\hline B10 & + & - & + & 0.00 & & \\
\hline B11 & + & - & - & 0.00 & & \\
\hline
\end{tabular}

Empty fields denote unsuccessful methylation readings. -, no expression; +, expression of genes in relation to the housekeeping gene Arp ${ }^{7}$.

$24.36 \%$ for dermoids). Although this suggests higher methylation in tumors, the difference was not statistically significant [Table I, $\mathrm{p}=0.06$ for fibromas and 0.31 for dermoids (Student's t-test)].

Methylation was detected in neither BCC nor normal skin samples in this region.

Region 3. This region $(-338$ to +135$)$ contains a transcription start, but it was not successfully amplified or sequenced in the selected samples. The unsuccessful analysis may perhaps be partly attributed to limited room for primers in the $\mathrm{CpG}$ dense environment (62 sites).

Region 4 containing ATG codon. The last part of the promoter, region $4(+126$ to +575 , with $\mathrm{ATG}$ at +373$)$ contains $66 \mathrm{CpG}$ islands. In this region prevalence of methylated $\mathrm{CpGs}$ in dermoids and fibromas relative to healthy ovary controls was seen by inspection, due to the very low methylation of normal tissue. Whereas dermoids and fibromas had on average $32.49 \%$ methylation of the successfully analyzed CpGs ( $38.49 \%$ for fibroma and $23.48 \%$ for dermoids), there was only $2.44 \%$ methylation in normal ovary samples. The Student's t-test gave a statistically significant difference, $\mathrm{p}=0.011$ for fibromas and 0.009 for dermoids.

Both BCC and normal skin samples showed extremely low methylation, with $<2 \%$ of methylated $\mathrm{CpGs}$ in both groups $(1.12 \%$ for BCC samples and $0.27 \%$ for healthy skin samples), $\mathrm{p}=0.091$ (Student's t-test) (Table I).

PTCH promoter mutations. No mutations or deletions were noted in promoter sequences during methylation analysis.

\section{Discussion}

The results of promoter methylation showed two different patterns. In basocellular carcinomas of the skin we observed no changes in the methylation status of the $\mathrm{PTCH}$ promoter in comparison to normal skin. It has been previously published that the whole PTCH pathway is activated in these types of tumors, and a high mutation rate of $P T C H$ was observed $(9,19)$. A large number of BCCs is associated with alterations in PTCH signaling, which is suggested as an option for therapeutic management of skin cancer (20).

Besides BCCs, our attention was on tumors where we have observed activation of the pathway, but without mutations in the PTCH gene, namely ovarian dermoids and fibromas. These two different patterns contribute to the existence of two independent tumor progression modes, with either promoter hypermethylation or a mutation of tumor suppressor PTCH as a way of pathway inactivation. While the role of PTCH in basocellular carcinomas was observed by others through mutational events of PTCH, SMO, SHH and even GLI1 (19), 
the methylation as an inactivating event was not known until now. Here we demonstrated that the promoter region of $P T C H$ in BCCs is not affected and therefore does not contribute to neoplastic phenotype.

Our results revealed significantly higher methylation of the $P T C H$ promoter region in ovarian tumor tissues than in normal ovarian tissue. This result was equally true for both fibroma and dermoid samples, just as was our previous finding of high $\mathrm{LOH}$ incidence at the PTCH locus in both tumor types (8). Furthermore, the tumors with LOH (samples F1 and F2) did not appear to differ significantly in methylation status from the others, suggesting that these two PTCH alterations happen independently.

Since negative results of the mutation search in its exons have narrowed the options of $P T C H$ involvement in these tumors to potential expression problems, our demonstration of methylated $\mathrm{CpG}$ sites in the promoter appears to be a viable indication of the $\mathrm{SHH} / \mathrm{PTCH}$ pathway malfunctioning in fibroma and dermoids.

Comparison with the expression pattern of the $\mathrm{SHH} /$ PTCH pathway genes in the same tumors may shed some light on the issue. The expression data published previously $(7,8)$ show moderately increased activities of various pathway genes. In particular, expression of GLI1 was not consistently increased in all samples, whereas almost all tumors showed some increase in both SHH and SMO expression $(7,8)$ which might in general be compatible with the low malignant potential of the analyzed tumor types; although details of the expression pattern did not quite fit into the typical model of the pathway activation). A similar expression analysis was performed here on BCC samples, and the results collated with expression data for dermoids and fibromas are reviewed in Table II. However, it should be understood that the expression data reflect the average activity of a tumor tissue slice, which may be lower (or different) than in the actual proliferating cells, as suggested by the Ptch immunostaining of dermoid lining (our previous results, 8).

Nevertheless, the compilation of our present and previous results in Table II may contribute to delineation of the potential role of $P T C H$ promoter methylation in the pathway malfunctioning. There were several cases of increased GLI expression (F1, F3, F6, D1, D2, D3; Table II), but PTCH did not appear to be consequently activated in either of them. The analysis of the promoter region 1 was successful in 8 cases, showing strong methylation, in particular of the $\mathrm{CpGs}$ in the vicinity of the Gli-binding sites. This is consistent with the hypothesis that methylation of the region prevents Gli protein from inducing transcription of the $P T C H$ gene. On the other hand, methylation of the promoter region 4 (containing the ATG codon) did not appear to have adverse effects on $P T C H$ activity, since the only two samples with high $P T C H$ expression $(\mathrm{F} 4, \mathrm{D} 4)$ both showed very strong methylation of this region.

Therefore, it seems likely that hypermethylation of the Gli-binding site on the $P T C H$ promoter (region 1) may cause or contribute to the tumor growth in some proportion of ovarian fibroma and dermoids, because it obstructs the pathway auto-regulatory loop in which the activated GLI1 stimulates expression of PTCH. As for the lack of detectable GLI1 activation in other samples, it has already been hypothesized that proliferation of these tumors is not necessarily always related to GLI activity (5).

Finally, it should be noted that $P T C H$ promoter hypermethylation is at best only one among many potential mechanisms of the pathway deregulation. Even when $\mathrm{PTCH}$ mutations are the main cause of deregulation, the alleles of this gene are frequently impaired by two unrelated events, as in BCNS tumors. A straightforward generalization would allow pathway deregulation by even less related genetic and/or epigenetic alterations: one of them may be insufficient synthesis of Ptch protein caused by methylated neighboring Gli1-binding sites (perhaps only on one PTCH promoter allele), whereas the others may include various (perhaps also hemizygous) aberrations of any pathway gene.

\section{Acknowledgements}

We are grateful to Oliver Vugrek and his group, Division of Molecular Medicine, Rudjer Boskovic Institute, Zagreb, Croatia, for help in the subcloning of PCR fragments. We thank D. Babic from the Department of Obstetrics and Gynecology, Clinical Hospital Petrova for the pathological analysis of samples. We thank K. Pavelic, the chairperson of the Division of Molecular Medicine in Rudjer Boskovic Institute for the enthusiasm and support. This study was supported by a grant of the Croatian Ministry of Science, Education and Sports, no. 14MP091.

\section{References}

1. Cohen MM Jr: The Hedgehog signaling network. Am J Med Genet 123A: 5-28, 2003.

2. Hahn H, Wicking C, Zaphiropoulous PG, Gailani MR, Shanley S, Chidambaram A, Vorechovsky I, Holmberg E, Unden AB, Gillies S, Negus K, Smyth I, et al: Mutations of the human homologue of Drosophila patched in the nevoid basal cell carcinoma syndrome. Cell 85: 841-851, 1996.

3. Levanat S, Gorlin RJ, Fallet S, Johnson DR, Fantasia JE and Bale AE: A two-hit model for developmental defects in Gorlin syndrome. Nat Genet 12: 85-87, 1996.

4. Agren M, Kogerman P, Kleman MI, Wessling M and Toftgard R: Expression of the PTCH1 tumor suppressor gene is regulated by alternative promoters and a single functional Gli-binding site. Gene 330: 101-114, 2004.

5. Ruiz I, Altaba A, Sanchez P and Dahmane N: Gli and hedgehog in cancer: tumours, embryos and stem cells. Nat Rev Cancer 2: 361-372, 2002.

6. Levanat S, Pavelic B, Crnic I, Oreskovic S and Manojlovic S: Involvment of PTCH gene in various noninflammatory cysts. J Mol Med 78: 140-146, 2000.

7. Levanat S, Musani V, Komar A and Oreskovic S: Role of the Hedgehog/Patched signaling pathway in oncogenesis. A new polymorphism in the PTCH gene in ovarian fibroma. Ann NY Acad Sci 1031: 134-143, 2004.

8. Levanat S, Kappler R, Hemmerlein B, Doring P, Musani V, Komar A, Oreskovic S, Paveliv B and Hahn H: Analysis of alterations of the PTCH1 signaling pathway in ovarian dermoids. Int J Mol Med 14: 793-799, 2004.

9. Daya-Grosjean L and Couve-Privat S: Sonic hedgehog signaling in basal cell carcinomas. Cancer Lett 225: 181-192, 2005.

10. Reik W, Dean W and Walter J: Epigenetic reprogramming in mammalian development. Science 293: 1089-1093, 2001.

11. Baylin SB: DNA methylation and gene silencing in cancer. Nat Clin Pract Oncol 2 (suppl 1): 4-11, 2005.

12. Baylin SB and Ohm JE: Epigenetic gene silencing in cancer - a mechanism for early oncogenic pathway addiction? Nat Rev Cancer 6: 107-116, 2006.

13. Feinberg AP, Ohlsson R and Henikoff S: The epigenetic progenitor origin of human cancer. Nat Rev Genet 7: 21-33, 2006 . 
14. Strathdee G, Appleton K, Illand M, Millan DW, Sargent J, Paul J and Brown R: Primary ovarian carcinomas display multiple methylator phenotypes involving known tumor suppressor genes. Am J Pathol 158: 1121-1127, 2001.

15. Frommer M, McDonald LE, Millar DS, Collis CM, Watt F, Grigg GW, Molloy PL and Paulet CL: A genomic sequencing protocol that yields a positive display of 5-methylcytosine residues in individual DNA strands. Proc Natl Acad Sci USA 89: 1827-1831, 1992.

16. Nagao K, Toyoda M, Takeuchi-Inoue K, Fujii K, Yamada M and Miyashita T: Identification and characterization of multiple isoforms of a murine and human tumor suppressor, patched, having distinct first exons. Genomics 85: 462-471, 2005.
17. Sanger F, Nicklen $S$ and Coulson AR: DNA sequencing with chain-terminating inhibitors. Proc Natl Acad Sci USA 74: 5463-5467, 1977.

18. Marchuk D, Drumm M, Saulino A and Collins F: Construction of T-vectors, a rapid and general system for direct cloning of unmodified PCR products. Nucleic Acids Res 19: 1154, 1991.

19. Lacour JP: Carcinogenesis of basal cell carcinomas: genetics and molecular mechanisms. Br J Dermatol 146 (suppl 61): 17-19, 2002.

20. Vogt A, Chuang PT, Hebert J, Hwang J, Lu Y, Kopelovich L, Athar M, Bickers DR and Epstein EH Jr: Immunoprevention of basal cell carcinomas with recombinant hedgehog-interacting protein. J Exp Med 199: 753-761, 2004. 